\title{
ECDC and WHO/Europe joint report on tuberculosis surveillance and monitoring in Europe
}

Eurosurveillance editorial team (eurosurveillance@ecdc.europa.eu) ${ }^{1}$

1. European Centre for Disease Prevention and Control (ECDC) Stockholm, Sweden

Citation style for this article:

Eurosurveillance editorial team. ECDC and WHO/Europe joint report on tuberculosis surveillance and monitoring in Europe . Euro Surveill. 2014;19(11):pii=20741.

Available online: http://www.eurosurveillance.org/ViewArticle.aspx?Articleld=20741

The European Centre for Disease Prevention and Control (ECDC) and the World Health Organization (WHO) Regional Office for Europe have jointly launched the sixth report on surveillance and monitoring of tuberculosis (TB) in Europe [1]. The report indicates that, in spite of notable progress in the past decade, TB is still a public health concern in many countries across Europe. High rates of multidrug-resistant (MDR) TB outside the European Union (EU)/European Economic Area (EEA) are of particular concern. Meanwhile EU/ EEA countries themselves have a significant number of TB cases among vulnerable population groups, such as people of foreign origin and prisoners.

In $2012,68,423$ cases of TB were reported in 29 EU/EEA countries, $6 \%$ less than in 2011, reflecting a decrease in 19 countries. The EU/EEA notification rate was 13.5 per 100,000 population. Eighty per cent of all notified TB cases were newly diagnosed and $70 \%$ of new pulmonary TB cases were culture-confirmed. Twentyseven per cent of all TB cases were of foreign origin, mostly residing in low-incidence countries. Adults were equally affected by TB, while the notification rate in children under the age of 15 years was 3.6 per 100,000, consistent with a slightly decreasing long-term trend. Males were over-represented in almost all EU/EEA Member States and among adults, with the greatest gender imbalance among those aged 45 to 64 years.
An assessment of progress towards TB elimination for the four epidemiological indicators and eight core indicators defined in the report 'Progressing towards TB elimination: A follow-up to the Framework Action Plan to Fight Tuberculosis in the European Union' [2] showed that none of the core indicators was achieved at EU/ EEA level.

Notwithstanding this, since 2001, TB incidence has been falling at an average rate of $5.0 \%$ per year, which is the fastest decline in the world.

ECDC and WHO/Europe have coordinated the collection and analysis of TB surveillance data across the countries of the WHO European Region (except Liechtenstein, Monaco and San Marino) since 2008.

References

1. European Centre for Disease Prevention and Control (ECDC) / World Health Organization (WHO) Regional Office for Europe. Surveillance report. Tuberculosis surveillance and monitoring in Europe 2014. Stockholm: ECDC; 2014. Available from: http://www.ecdc.europa.eu/en/publications/Publications/ tuberculosis-surveillance-monitoring-Europe-2014.pdf

2. European Centre for Disease Prevention and Control (ECDC). Progressing towards TB elimination: A follow-up to the Framework Action Plan to Fight Tuberculosis in the European Union. Stockholm: ECDC; 2010. Available from: http:// ecdc.europa.eu/en/publications/publications/101111 spr progressing_towards_tb_elimination.pdf 